\title{
Future Skills für die Welt von morgen: Das Future-Skills-Triple-Helix-Modell der Handlungsfähigkeit in emergenten Praxiskontexten
}

\section{Ulf-Daniel Ehlers}

\section{Zusammenfassung}

In einer Welt im immer schnelleren Wandel ist die Diskussion um Future Skills eine der aktuellsten in der Bildungsforschung. Dabei wird die Diskussion um Future Skills schon lange geführt (angefangen mit Studien zu Graduate Attributes), ist oft ungreifbar wegen konzeptioneller Unklarheit darüber, was Skills eigentlich sind, und bezieht sich oft nur verkürzt auf digitale Future Skills. Die hier dargestellte Forschung basiert auf einem fundierten empirischen Ansatz, den multimethodischen angelegten, mehrteiligen NextSkills-Studien. Die Intention des Projekts besteht darin, die Nachfrage nach spezifischen Future Skills näher zu erforschen und diese dann im zweiten Schritt auch bildungstheoretisch zu fundieren. Diese Future Skills werden durch das „Future-Skills-Triple-Helix-Modell der Handlungsfähigkeit in emergenten Praxiskontexten" eingeordnet. Im Einzelnen handelt es sich um Skills, wie beispielsweise Ambiguitätskompetenz, ethische Kompetenz, Selbstkompetenz und anderes.

\section{Schlüsselwörter}

Hochschulwandel • Future Skills • Triple Helix • Bildungsforschung • Zukunftsfähigkeit • Kompetenzentwicklung • Delphi-Studie

U.-D. Ehlers $(\bowtie)$

Duale Hochschule Baden-Württemberg, Karlsruhe, Deutschland E-Mail: ulf-daniel.ehlers@dhbw-karlsruhe.de 


\section{Future Skills - Leitmarken einer neuen Bildungskonzeption für Hochschulen}

Im Beitrag definieren wir Future Skills als Kompetenzen, die es Individuen erlauben, in hochemergenten Handlungskontexten selbst organisiert komplexe Probleme zu lösen und (erfolgreich) handlungsfähig zu sein. Sie basieren auf kognitiven, motivationalen, volitionalen sowie sozialen Ressourcen, sind wertebasiert und können in einem Lernprozess angeeignet werden. In der öffentlichen Diskussion über Hochschulbildungskonzepte haben sie mittlerweile zu einem entscheidenden Wandel beigetragen, den wir hier als Future Skills Turn bezeichnen (Ehlers 2020a). Diesen aufzuarbeiten und in seiner Tragweite für die Konzeption künftiger Hochschulbildung zu erfassen ist der Gegenstand der hier dargestellten Forschung. Als Begriff hat Future Skills einen Einfluss gewonnen, wie er in den Siebzigerjahren des letzten Jahrhunderts von Begriffen wie Chancengleichheit oder Wissenschaftsorientierung ausgegangen ist. Solche Leitmarken treten nicht als exakt zugeschnittene und empirisch operationalisierte Konzepte auf, sondern viel eher als begriffliche Verdichtungen breit gefächerter Bündel von Argumenten und Zielsetzungen (Packe und Schleiermacher 2018; Ehlers 2020a).

Ausgangspunkt für die enorme Karriere des Konzeptes der Future Skills ist die Diagnose, dass derzeitige Konzepte der Hochschulbildung den drängenden Herausforderungen unserer Gesellschaften keine überzeugenden Zukunftskonzepte entgegenstellen (Hippler 2016; Kummert 2017) - weder der nachhaltigen Gestaltung unserer Umwelt noch den damit zusammenhängenden sozialen oder ökonomischen Herausforderungen. Während die gesellschaftlichen Problemlagen von einem sich stetig beschleunigenden Globalisierungsprozess und einem immer schneller werdenden digitalen Fortschritt verschärft werden, liegen genau hier auch die Kräfte der Ermöglichung einer Vielzahl neuer Optionen für die menschliche Entwicklung. In dieser Situation digitaler Beschleunigung ist das kennzeichnende Merkmal das der Unsicherheit und die unausweichliche Notwendigkeit die der Gestaltungsverantwortung. Denn die Zukunft ist unvorhersehbar und wir können sie nicht prognostizieren, müssen aber bereit sein, sie zu gestalten.

Kinder, die im nächsten Jahr in die Grundschulen kommen, werden in zehn bis zwölf Jahren in eine Berufsausbildung oder ein Studium gehen und in fünfzehn Jahren diejenigen sein, die als junge Berufstätige beginnen, unsere Gesellschaft zu prägen. Über diese Zukunft wissen wir wenig. In den Jahren 2060-2065 werden sie aller Voraussicht nach ihre Erwerbstätigkeit beenden. Über diese Zukunft wissen wir nichts. Unsere Schulen müssen sie auf Jobs vorbereiten, die es heute noch nicht gibt, auf Technologien, Apps und Anwendungen, die heute noch nicht erfunden worden sind, darauf, in einer Gesellschaft zu leben, deren Strukturen wir 
heute nicht absehen können, und mit Herausforderungen umzugehen, die heute noch nicht erkennbar sind. Es ist unser aller gemeinsame Verantwortung, das Beste aus den Möglichkeiten zu machen und Wege zu finden, mit dieser ungewissen Zukunft umzugehen. Dabei geht es um nicht mehr und nicht weniger als den Erhalt unseres Planeten und unserer Lebensgrundlagen.

Das Lösen der gesellschaftlichen Problemlagen, wie sie etwa mit dem Klimawandel verbunden sind, der Herausforderungen der zukünftig noch zunehmenden Migration, der Konflikte, die durch populistische Gesellschafts- und Politikentwürfe entstehen und der damit verbundenen Frage nach der Zukunft der Demokratie - all dies erfordert die Fähigkeit, neue und bisher unbekannte Ansätze zu entwickeln, neue Wege zu gehen und bislang Unverbundenes auf neue Weise miteinander in Beziehung zu setzen. In der Bildung und Wissenschaft wird dies nur dann gelingen, wenn wir im besten Sinne inter- und transdisziplinär daran arbeiten, die Lösungsbeiträge einer jeden Disziplin und Wissenschaft zusammenzutragen, kritisch zu reflektieren und aufeinander zu beziehen. Hochschulen tun sich dabei schwer - denn sie alle teilen ein gemeinsames Handicap: Die Geschichte der Wissenschaft, Forschung und damit auch der Hochschulbildung ist eine Geschichte der Differenzierung, Spezialisierung und Abgrenzung der Disziplinen. Die fast 18.000 Studiengänge, die an deutschen Hochschulen angeboten werden, zeugen davon (Hachmeister 2017). Die Institution Hochschule steht vor der Herausforderung, sich selbst neu zu erfinden - und das in einer Zeit, in der sie sich in einem enormen Wachstumsprozess befindet und weltweit eine Quote von $70 \%$ Studierenden einer Alterskohorte oder mehr bis ins Jahr 2050 prognostiziert wird. Das ist in etwa so, als müsse man bei einem Autorennen, mitten in der Steilkurve und während eines gefährlichen Überholmanövers die Pilotin oder den Piloten wechseln. Die Hochschule muss sich damit auseinandersetzen, welche Future Skills es sind, die die Absolvent*innen von morgen benötigen, und wie sie sie bei deren Erwerb unterstützen kann. Dafür gilt es zunächst, diese Future Skills bildungstheoretisch zu beschreiben - und dies kann durch das Future-Skills-Triple-Helix-Modell geschehen, welches im Rahmen der NextSkills-Studien (www.nextskills.org) entwickelt werden konnte (Ehlers 2020b).

\section{Forschungsdesign}

Das Forschungsvorhaben NextSkills zielt darauf ab zu analysieren, welche Fähigkeiten für eine produktive und proaktive Gestaltung zukünftiger Arbeitskontexte 
benötigt werden, um Anforderungen an Hochschulen abzuleiten. Dazu wurden in einem mehrschrittigen Forschungsprozess Future-Skill-Profile ermittelt:

1. Identifikation von Future Organisations: In einem ersten Schritt wurden Organisationen identifiziert, die bereits explizite Erfahrungen bei der Implementierung von Kompetenzmodellen, Vorstellung über Future Skills und einen hohen Reifegrad bei der Gestaltung zukünftiger Arbeitskontexte hatten. Dazu wurden sogenannte Future Organisations identifiziert, die als empirisches Feld geeignete Kontexte zur Ermittlung von Future Skills ausgeprägt haben. Das Auswahlverfahren fand im Jahr 2015 im Rahmen eines Wettbewerbs statt, bei dem über 8500 Partnerorganisationen der Dualen Hochschule BadenWürttemberg angeschrieben wurden und sie die Möglichkeit hatten, ihre Personalentwicklungs- und insbesondere ihre Konzeptionen für die Betreuung und Förderung von Studierenden einzureichen. An dem Wettbewerb ${ }^{1}$ beteiligten sich 124 Organisationen. Alle eingereichten Konzeptionen wurden in einem kriteriengestützten Expert*innenenrating bewertet. Das so entstehende Ranking wurde dann bei einer Diskussion von 15 Expert*innen diskursiv validiert und 20 Organisationen und ihre Kompetenzkonzeptionen wurden für eine Shortlist ausgewählt. Alle 20 Organisationen wurden eingeladen, am nächsten Schritt der NextSkills-Studie teilzunehmen, 17 reagierten positiv und wurden in die Interviewstudie mitaufgenommen. Die Interviews fanden zwischen Dezember 2016 und Juni 2017 statt.

2. Interviewstudie: Für die Interviewstudie wurden Leitfragen entwickelt, die zur Orientierung im Rahmen eines offenen, wenig strukturierten, problemvertiefenden Interviews eingesetzt wurden. Teilnehmende der Interviews waren die Personalverantwortlichen der Organisationen und teilweise auch die Studierenden, die im Rahmen von (dualen) Studiengängen dort studierten. Insgesamt wurden 17 vertiefende Interviews geführt, an denen sich 20 Personen beteiligten und die zu etwa 700 min qualitativem Interviewmaterial führten. Die Interviews wurden wortgetreu transkribiert und unter Anwendung der induktiven Kodierungstechnik (Mayring 1996; Thomas 2006) mithilfe der Software MaxQDA von zwei Forschenden unabhängig kodiert. Konstrukte wurden aus den Interviewdaten herausgearbeitet, um Kontexte, Werte sowie Abläufe und Abhängigkeiten für zukünftig als wichtig erachtete Fähigkeiten bei Individuen zu rekonstruieren.

\footnotetext{
${ }^{1}$ Der Wettbewerb war in Kooperation der Dualen Hochschule Baden-Württemberg, des Ministeriums für Wissenschaft, Kunst und Kultur Baden-Württemberg und des Arbeitgeberverbands Südwestmetall organisiert und als „Dualer Partner Award“ ausgeschrieben worden.
} 
3. Internationale Delphi-Studie: Um die qualitativ erworbenen Ergebnisse weiter zu verfeinern und zu validieren, wurde eine Delphi-Studie mit einem internationalen Expert*innenpanel durchgeführt. Die Delphi-Studie mit dem Titel „Future Skills - Future Learning and Future Higher Education“ (Ehlers und Kellermann 2019) umfasste zwei Befragungsrunden. Zur Studie wurden 53 internationale Expert*innen aus unterschiedlichen Organisationen und Institutionen zur Teilnahme eingeladen (ebenda).

\section{$3 \quad$ Future Skills für die Welt von morgen}

Die Hochschulbildung der Zukunft muss an der Vermittlung von Future Skills orientiert sein. Das zeigen die Ergebnisse der NextSkills-Studie. Auf Basis der Tiefeninterviews und durch Einschätzung der weltweit befragten Expertinnen und Experten wurden 17 Skill-Profile konstruiert, die für zukünftige Hochschulabsolvent*innen Bedeutung haben. Jedes Skill-Profil besteht aus einem Bündel einzelner Kompetenzen, sogenannter Bezugskompetenzen. Skill-Profile sind gleichsam Cluster von zukunftsrelevanten Fähigkeiten. Sie sind wiederum in drei sogenannte Kompetenzfelder eingeteilt.

Zugleich bildet die Studie die empirische Grundlage, auf der das Triple-HelixModell der Handlungsfähigkeit in emergenten Praxiskontexten konstruiert wurde. Future Skills sind Teil der Kompetenzwende, des Future Skills Turn, der an den Hochschulen der Zukunft notwendig ist. Sie markieren einen Turn zu einer Hochschulbildung, die nicht mehr die Funktion der Vorbereitung durch Wissenstransfer in den Mittelpunkt stellt, sondern die Studierende bei der Entwicklung von Future Skills, also Handlungsdispositionen und Handlungsbereitschaft für den Umgang mit komplexen, unbekannten Problemsituationen durch Reflexion, Werte und Haltungen, unterstützt. Future Skills sind dabei wie folgt definiert:

Future Skills sind Kompetenzen, die es Individuen erlauben, in hochemergenten Handlungskontexten selbst organisiert komplexe Probleme zu lösen und (erfolgreich) handlungsfähig zu sein. Sie basieren auf kognitiven, motivationalen, volitionalen sowie sozialen Ressourcen, sind wertebasiert und können in einem Lernprozess angeeignet werden.

Formuliert man Future Skills kompetenztheoretisch, so wird deutlich, dass es sich bei ihnen um Kompetenzkonstrukte mit besonderer inhaltlicher Profilierung handelt (Abb. 1). Sie ermöglichen es Individuen, in hochemergenten Kontexten zu handeln. Aus kompetenztheoretischer Perspektive kommt dabei die Fähigkeit zum 


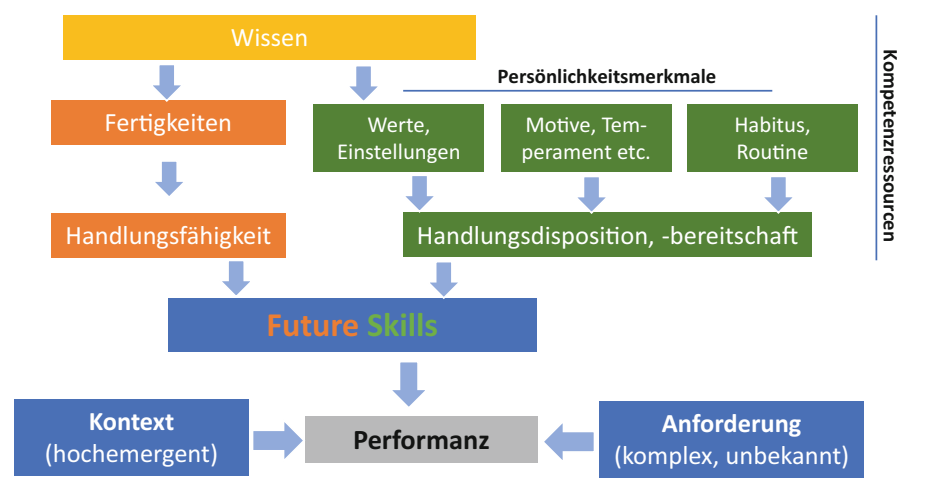

Abb. 1 Das Future-Skills-Konzept als Kompetenzkonstrukt. (Eigene Darstellung)

Handeln (gespeist aus Wissen und weiterentwickelt zu Fertigkeiten) mit Handlungsdispositionen und -bereitschaft zusammen, die sich vor allem aus Werten, motivationalen und habituellen Faktoren speist - also Persönlichkeitsmerkmalen.

Im Begriff und im Konzept lassen sich Future Skills von solchen Kompetenzen abgrenzen, die nicht im besonderen Maße zukunftsorientiert sind. Als Unterscheidungsdimension zwischen aktuellen oder bisherigen Kompetenzanforderungen und solchen, die zukunftsrelevant sind, gilt dabei das Konzept der Emergenz: Insbesondere solche Handlungskontexte, die hochemergente Entwicklungen von Lebens-, Arbeits-, Organisations- und Geschäftsprozessen aufweisen, benötigen Future Skills zur Bewältigung der Anforderungen. Emergenz definiert also die Trennlinie, die bisherige oder traditionelle Arbeitsbereiche und zukünftige Arbeitsbereiche voneinander abgrenzt. Da diese Grenze nicht klar schematisch, sondern fließend verläuft und viele Organisationen sich in Transformationsprozessen befinden, in denen sich schwach emergente Arbeitskontexte zu hochemergenten Arbeitskontexten entwickeln, ist auch die Notwendigkeit von Future Skills ein sich entwickelnder Bereich und nicht ein binärer Zustand des Entweder-oder.

Emergenz versus Submergenz ist also eine wichtige Grundunterscheidung zur Erklärung der Bedeutung von Future Skills. Die NextSkills-Studie zeigt, dass niedrigemergente (stabile) professionelle Handlungskontexte sich oft, schnell und in hoher Intensität hin zu hochemergenten Handlungskontexten wandeln. Wir sprechen hier von der Drift-to-Self-Organisation. Dieser Wandel entspricht einer Veränderung im Systemzustand von Organisationen. Er wird durch Veränderungen von Makro-, Meso- und Mikrosystemen ausgelöst und durch deren 
interdependente Verschränkung verstärkt. Im dadurch entstehenden neuen Systemzustand lassen sich die Systemelemente nicht kausal oder linear auf den vorherigen Zustand zurückführen. Es gilt die Systembedingung der Irreduzibilität sowie der Unvorhersagbarkeit.

Die in der NextSkills-Studie auf Basis von Tiefeninterviews rekonstruierten Future-Skills-Profile sind in Tab. 1 überblicksartig dargestellt. FutureSkills-Profile bestehen aus Bündeln einzelner zusammengehörender sogenannten Bezugskompetenzen. Insgesamt lassen sich aus den qualitativen Daten 17 solcher Kompetenzprofile rekonstruieren (siehe Abb. 2), die im Folgenden dargestellt und beschrieben werden. Sie sind in die drei Kompetenzfelder des Triple-HelixModells gegliedert.

Die Einteilung in drei Kompetenzfelder, die in der Grafik als drei Straßenbahnlinien der Future Skills Map dargestellt sind, folgt der Systematik des Triple-Helix-Modells für Future Skills. Sie basiert auf der Erkenntnis, dass die zur Bewältigung von Handlungsanforderungen notwendigen Skills sich anhand von drei zusammenwirkenden Dimensionen strukturieren lassen, die im TripleHelix-Modell mit spezifischen Begriffen bezeichnet werden:

1. Individuell-entwicklungsbezogene Future Skills, die sich auf die Entwicklungsfähigkeit der eigenen Person beziehen, hier individuellentwicklungsbezogene Kompetenzen genannt,

2. solche Future Skills, die sich auf den Umgang mit bestimmten Gegenständen, Arbeitsaufgaben und Problemstellungen beziehen, hier individuellobjektbezogene Kompetenzen genannt, und

3. solche Future Skills, die sich auf den Umgang mit der sozialen, organisationalen und institutionellen Umwelt beziehen, hier als organisationsbezogene Kompetenzen bezeichnet.

Innerhalb dieses dreidimensionalen Handlungsraumes können die von den Befragten genannten einzelnen Future Skills konzeptionell verortet werden.

\section{Future-Skill-Profile der NextSkills-Studie}

Tab. 1 stellt die einzelnen Future-Skill-Profile, dazugehörige Bezugskompetenzen sowie die Beschreibungen der Kompetenzfelder noch einmal im Überblick dar. 
Tab. 1 Future-Skills-Kompetenzfelder und -profile im Überblick. (Eigene Darstellung)

\begin{tabular}{|c|c|c|}
\hline ID & $\begin{array}{l}\text { Kompetenzfeld/Future- } \\
\text { Skill-Profill } \\
\text { Bezugskompetenzen }\end{array}$ & Beschreibung \\
\hline I & $\begin{array}{l}\text { Subjekt- } \\
\text { entwicklungsbezogene } \\
\text { Kompetenzen }\end{array}$ & $\begin{array}{l}\text { Subjekt-entwicklungsbezogene Kompetenzen umfassen die Fähigkeiten, im } \\
\text { eigenen Professionsumfeld subjektiv handlungsfähig und aus sich heraus, } \\
\text { selbst gesteuert zu lernen und sich zu entwickeln. Dabei spielen eine hohe } \\
\text { Autonomie, Selbstkompetenz, Selbstwirksamkeit und Leistungsmotivation } \\
\text { eine wichtige Rolle. }\end{array}$ \\
\hline 1 & Lernkompetenz & $\begin{array}{l}\text { Lernkompetenz ist die Fähigkeit und Bereitschaft zum Lernen, insbesondere zum } \\
\text { selbst gesteuerten Lernen. Sie erstreckt sich auch auf metakognitive Fähigkeiten. }\end{array}$ \\
\hline 2 & Selbstwirksamkeit & $\begin{array}{l}\text { Selbstwirksamkeit ist die Überzeugung und das (Selbst-)Bewusstsein dafür, die zu } \\
\text { bewältigenden Aufgaben mit den eignen Fähigkeiten umsetzen zu können, dabei } \\
\text { Verantwortung zu übernehmen und Entscheidungen treffen zu können. }\end{array}$ \\
\hline 3 & $\begin{array}{l}\text { Selbstbestimmungskompe- } \\
\text { tenz }\end{array}$ & $\begin{array}{l}\text { Selbstbestimmungskompetenz bezeichnet die Fähigkeit, im Spannungsverhältnis } \\
\text { von Fremd- und Selbstbestimmung produktiv zu agieren und sich Räume zur } \\
\text { eigenen Autonomie und Entwicklung zu schaffen, sodass die Befriedigung der } \\
\text { eigenen Bedürfnisse in Freiheit und selbstbestimmt angestrebt werden kann. }\end{array}$ \\
\hline 4 & Selbstkompetenz & $\begin{array}{l}\text { Selbstkompetenz ist die Fähigkeit, die eigene persönliche und berufliche } \\
\text { Entwicklung weitgehend unabhängig von äußeren Einflüssen zu gestalten. Dazu } \\
\text { gehören Teilkompetenzen wie zum Beispiel selbstständige Motivation, Zielsetzung, } \\
\text { Planung, Zeitmanagement, Organisation, Lernfähigkeit und Erfolgskontrolle durch } \\
\text { Feedback, aber auch Cognitive Load Management und eine hohe } \\
\text { Eigenverantwortlichkeit. }\end{array}$ \\
\hline 5 & Reflexionskompetenz & $\begin{array}{l}\text { Reflexionskompetenz umfasst die Bereitschaft und Fähigkeit zur Reflexion, also die } \\
\text { Fähigkeit, sich selbst und andere zum Zweck der konstruktiven Weiterentwicklung } \\
\text { zu hinterfragen sowie zugrundeliegende Verhaltens-, Denk- und Wertesysteme zu } \\
\text { erkennen und deren Konsequenzen für Handlungen und Entscheidungen holistisch } \\
\text { einzuschätzen. }\end{array}$ \\
\hline 6 & Entscheidungskompetenz & $\begin{array}{l}\text { Entscheidungskompetenz ist die Fähigkeit, Entscheidungsnotwendigkeiten } \\
\text { wahrzunehmen sowie mögliche alternative Entscheidungen gegeneinander } \\
\text { abzuwägen, eine Entscheidung zu treffen und diese auch zu verantworten. }\end{array}$ \\
\hline 7 & $\begin{array}{l}\text { Initiativ- und } \\
\text { Leistungskompetenz }\end{array}$ & $\begin{array}{l}\text { Initiativ- und Leistungskompetenz ist die Fähigkeit zur Selbstmotivation sowie der } \\
\text { Wunsch, etwas beizutragen. Beharrlichkeit und Zielorientierung formen die } \\
\text { Leistungsmotivation. Zusätzlich spielt ein positives Selbstkonzept eine Rolle, sodass } \\
\text { Erfolge und Misserfolge in einer Weise attribuiert werden, die nicht zur Senkung der } \\
\text { Leistungsmotivation führen. }\end{array}$ \\
\hline 8 & Ambiguitätskompetenz & $\begin{array}{l}\text { Ambiguitätskompetenz ist die Fähigkeit, Vieldeutigkeit, Heterogenität und } \\
\text { Unsicherheit zu erkennen, zu verstehen und produktiv gestaltend damit zu umgehen } \\
\text { sowie in unterschiedlichen Rollen zu agieren. }\end{array}$ \\
\hline 9 & Ethische Kompetenz & $\begin{array}{l}\text { Ethische Kompetenz umfasst die Fähigkeit zur Wahrnehmung eines Sachverhalts } \\
\text { beziehungsweise einer Situation als ethisch relevant einschließlich ihrer } \\
\text { begrifflichen, empirischen und kontextuellen Prüfung (wahrnehmen), die Fähigkeit } \\
\text { zur Formulierung von einschlägigen präskriptiven Prämissen zusammen mit der } \\
\text { Prüfung ihrer Einschlägigkeit, ihres Gewichts, ihrer Begründung, ihrer } \\
\text { Verbindlichkeit und ihrer Anwendungsbedingungen (bewerten) sowie die Fähigkeit } \\
\text { zur Urteilsbildung und der Prüfung ihrer logischen Konsistenz, ihrer } \\
\text { Anwendungsbedingungen und ihrer Alternativen (urteilen). }\end{array}$ \\
\hline II & $\begin{array}{l}\text { Individuell- } \\
\text { objektbezogene } \\
\text { Kompetenzen }\end{array}$ & $\begin{array}{l}\text { In einer zweiten Gruppe von Kompetenzen befinden sich sogenannte } \\
\text { individuell-objektbezogene Fähigkeiten. Dies sind Fähigkeiten in Bezug auf } \\
\text { bestimmte Gegenstände, Themen und Aufgabenstellungen kreativ, agil, } \\
\text { analytisch und mit hohem Systemverständnis zu agieren, auch unter } \\
\text { hochgradig unsicheren und unbekannten Bedingungen. }\end{array}$ \\
\hline 10 & $\begin{array}{l}\text { Design-Thinking- } \\
\text { Kompetenz }\end{array}$ & $\begin{array}{l}\text { Design-Thinking-Kompetenz ist die Fähigkeit, in einem gegebenen Kontext und in } \\
\text { Bezug auf einen bestimmten gegebenen Gegenstand (Objekt) kreativ } \\
\text { Veränderungen anzustreben, Rahmenbedingungen und Anforderungen des } \\
\text { jeweiligen Kontexts wahrzunehmen und zu analysieren, daraus Ideen zu generieren } \\
\text { und Handlungen abzuleiten. Dabei spielen Interdisziplinarität, die Fähigkeit zum } \\
\text { Perspektivwechsel und Flexibilität in der Lösungssuche sowie Offenheit } \\
\text { verschiedenen Ansätzen gegenüber eine besonders wichtige Rolle. }\end{array}$ \\
\hline 11 & Innovationskompetenz & $\begin{array}{l}\text { Innovationskompetenz ist die Fähigkeit und Bereitschaft, zu experimentieren und } \\
\text { dabei kreativ Neues und vorher Unbekanntes zu schaffen, indem Assoziation, } \\
\text { Dekonstruktion und Konstruktion genutzt werden. }\end{array}$ \\
\hline
\end{tabular}


Tab. 1 (Fortsetzung)

\begin{tabular}{|c|c|c|}
\hline 12 & Systemkompetenz & $\begin{array}{l}\text { Systemkompetenz ist die Fähigkeit und Bereitschaft, einzelne Phänomene als } \\
\text { einem größeren System zugehörig zu erkennen, Systemgrenzen und Teilsysteme } \\
\text { sowohl zu identifizieren als auch sinnvoll zu bilden, die Funktionsweise von } \\
\text { Systemen zu verstehen und aufgrund der Kenntnis der Veränderungen einzelner } \\
\text { Systemkomponenten Vorhersagen über die weitere Entwicklung des Systems zu } \\
\text { machen sowie sie in verschiedenen Situationen und Kontexten umzusetzen und } \\
\text { anzuwenden. Dazu gehört auch die Fähigkeit, sich an Systembedingungen } \\
\text { anzupassen, um in einem System in gewünschtem Maße agieren zu können. }\end{array}$ \\
\hline 13 & Digitalkompetenz & $\begin{array}{l}\text { Digitalkompetenz ist die Fähigkeit, digitale Medien zu nutzen, produktiv gestaltend } \\
\text { zu entwickeln, für das eigene Leben einzusetzen und reflektorisch-analytisch ihre } \\
\text { Wirkungsweise zu verstehen sowie die Potenziale und Grenzen digitaler Medien } \\
\text { und ihrer Wirkungsweisen einzuschätzen. }\end{array}$ \\
\hline III & $\begin{array}{l}\text { Organisationsbezogene } \\
\text { Kompetenzen }\end{array}$ & $\begin{array}{l}\text { In einer dritten Gruppe befinden sich Kompetenzen, die sich auf den Umgang } \\
\text { mit der sozialen, organisationalen und institutionellen Umwelt beziehen. } \\
\text { Hierzu gehören Fähigkeiten wie Sinnstiftung und Wertebezogenheit, die } \\
\text { Fähigkeit, Zukunft gestaltend mitzubestimmen, mit anderen } \\
\text { zusammenzuarbeiten und zu kooperieren und in besonderer Weise } \\
\text { kommunikationsfähig, kritik- und konsensfähig zu sein. }\end{array}$ \\
\hline 14 & Sensemaking & $\begin{array}{l}\text { Sensemaking (Sinnstiftung) beschreibt den Prozess, mit dem Menschen den über } \\
\text { die Sinne ungegliedert aufgenommenen Erlebnisstrom in sinnvolle Einheiten } \\
\text { einordnen. Je nach Einordnung der Erfahrung kann sich ein unterschiedlicher Sinn } \\
\text { und damit eine andere Erklärung für die aufgenommenen Erlebnisse ergeben. Es } \\
\text { ist insbesondere die Fähigkeit, in unterschiedlichen (organisationalen) Kontexten } \\
\text { einerseits Strukturen und Werte zu erkennen und andererseits Erfahrungen und } \\
\text { Wahrnehmungen produktiv und positiv in für sich sinnvolle Bedeutungen zu } \\
\text { gliedern. }\end{array}$ \\
\hline 15 & $\begin{array}{l}\text { Zukunfts- und } \\
\text { Gestaltungskompetenz }\end{array}$ & $\begin{array}{l}\text { Zukunftskompetenz ist die Fähigkeit, mit Mut zum Neuen, Veränderungsbereitschaft } \\
\text { und Vorwärtsgewandtheit die derzeit gegebenen Situationen in andere, neue und } \\
\text { bisher nicht bekannte Zukunftsvorstellungen weiterzuentwickeln und diese } \\
\text { gestalterisch anzugehen. }\end{array}$ \\
\hline 16 & Kooperationskompetenz & $\begin{array}{l}\text { Kooperationskompetenz ist die Fähigkeit zur Zusammenarbeit in Teams, auch } \\
\text { interkulturell, in Präsenzinteraktion oder unter Zuhilfenahme von Medien, innerhalb } \\
\text { oder zwischen Organisationen Zusammenarbeit so zu gestalten, dass bestehende } \\
\text { Differenzen in Gemeinsamkeiten überführt werden können. Dabei spielen soziale } \\
\text { Intelligenz, Offenheit und Beratungskompetenz eine wichtige Rolle. }\end{array}$ \\
\hline 17 & Kommunikationskompetenz & $\begin{array}{l}\text { Kommunikationskompetenz umfasst neben sprachlichen Fähigkeiten auch Diskurs-, } \\
\text { Dialog- und strategische Kommunikationsfähigkeit, um in unterschiedlichen } \\
\text { Kontexten und Situationen situativ angemessen erfolgreich kommunikativ } \\
\text { handlungsfähig zu sein. }\end{array}$ \\
\hline
\end{tabular}

\section{Das Future-Skills-Triple-Helix-Modell}

Future Skills können hinsichtlich ihrer Binnenstruktur noch weiter unterteilt werden. Dazu muss zunächst angemerkt werden, dass Skill ein Terminus ist, der immer eine Relation zwischen einem (Anforderungs-)Kontext einerseits und einer Handlung andererseits ausdrückt. In Ehlers (2020a) wird ausgeführt, dass sich in den empirischen Daten der Future-Skills-Studie nicht eine, sondern drei solcher Relationen rekonstruieren lassen: Eine handelnde Person kann Future Skills in Bezug auf sich selbst entwickeln, kann sie in Bezug auf den Umgang mit einer Aufgabe, einem Thema oder einem Gegenstand, den sie bearbeitet, entwickeln oder in Bezug auf die organisationale Umwelt, also das soziale System 

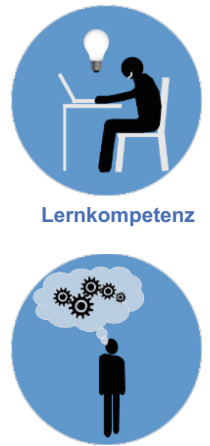

Reflexionskompetenz

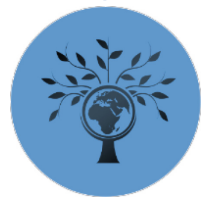

Ethische Kompetenz

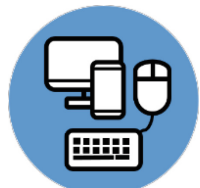

Digitalkompetenz

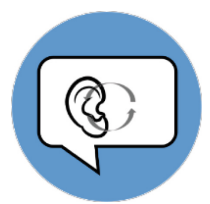

Kommunikationskompetenz

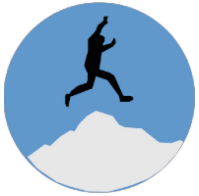

Selbstwirksamkeit

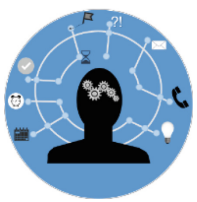

Entscheidungskompetenz

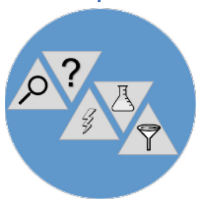

Design-Thinking Kompetenz

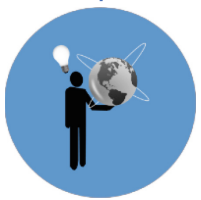

Sensemaking

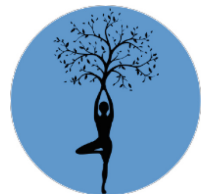

Selbstbestimmungskompetenz

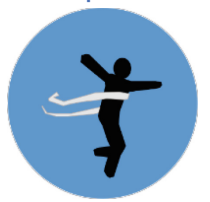

Initiativ- und Leistungskompetenz

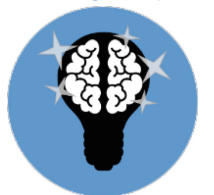

Innovationskompetenz

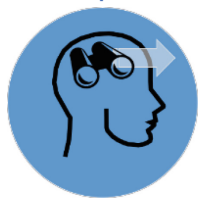

Zukunfts- und Gestaltungskompetenz

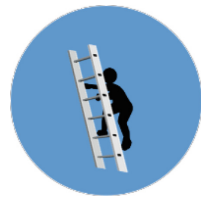

Selbstkompetenz

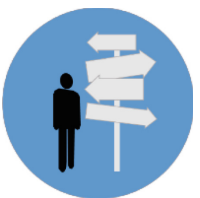

Ambiguitäts kompetenz

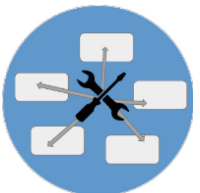

Systemkompetenz

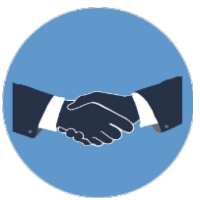

Kooperationskompetenz

Abb. 2 Future-Skill-Profile im Überblick. (Eigene Darstellung)

(zur Detaildarstellung der zugrunde liegenden erkenntnistheoretischen Position siehe auch Ehlers 2020a). In der Rekonstruktion der Daten benennen wir diese 
drei Relationen als Subjekt-, Objekt- und (sozialen/organisationalen) Weltbezug. $^{2}$ Es entsteht eine dreipolige Relation, wobei jeder Pol in Relation zum jeweils anderen steht. In Bezug auf Handlungen in hochemergenten Kontexten sind damit in jeder beliebigen Handlung immer alle drei Pole und deren Beziehung untereinander bestimmend. Aufgrund der engen Verwobenheit aller drei Pole und ihrer aufeinander bezogenen Integration bezeichnen wir dieses Konzept als Future-Skills-Triple-Helix-Modell. Das so entstehende Konzept ist geeignet zur formalen Beschreibung von Handlungen in hochemergenten Kontexten. In Beantwortung der Frage, ob es sich eher um eine subjektive, auf sich selbst bezogene Fähigkeit (beispielsweise selbst gesteuertes Lernen, Selbstkompetenz), eine auf einen Gegenstand oder eine Aufgabe bezogene Fähigkeit oder um eine auf die soziale, organisationale Umwelt bezogene Fähigkeit handelt, lassen sich die Future-Skill-Konstrukte in drei Bereiche einteilen und binnendifferenzieren. Als Klassifizierungskriterium dient dabei das Ziel der Relation - ob es auf ein Subjekt (Individuum zu sich selbst), Objekt (Individuum zu einem bestimmten Objekt, beispielsweise einer Aufgabe) oder die Umwelt (Individuum zur sozialen Umwelt) bezogen ist:

1. Beziehung eines Individuums zu sich selbst in der Gegenwart, Vergangenheit oder Zukunft (Subjekt- oder Zeitdimension), ${ }^{3}$

2. Beziehung eines Individuums zu einem bestimmten Objekt (Objektdimension) oder

3. Beziehung eines Individuums zu einer Person oder einer Gruppe in der Welt (soziale Dimension).

Diese dreigliedrige Unterteilung ist in der Philosophie der Bildungswissenschaften tief verankert (zum Beispiel Dewey und Bentley in ihrem Aufsatz Knowing the Known Dewey und Bentley 1949), geht in der Aktualität aber maßgeblich auf Meder (2007; auch Roth 1971) zurück, der eine fundamentale, konstitutive Struktur für Bildung als einer strukturell-dreigliedrigen Beziehung aufstellt. Für das Future-Skills-Konzept ergibt sich daraus eine dreidimensionale Aufgliederung:

\footnotetext{
${ }^{2}$ Die ,Subjekt-Objekt-Spaltung“ bezieht sich auf eine erkenntnistheoretische Grundstruktur, die damit gegeben ist, dass sich das menschliche Bewusstsein auf Gegenstände bezieht. Zwischen Erkenntnisgegenstand (Objekt) und Erkennendem (Subjekt) besteht stets eine unaufhebbare Differenz. Dies gelte auch für den Fall, dass das Ich sich selbst reflektiert, also für gegenständliches Selbstbewusstsein.

${ }^{3}$ Der Begriff Zeitdimension geht darauf zurück, dass sich Subjekte nur in der Zeit wahrnehmen können, also in Bezug auf etwas Vergangenes, etwas gerade Passierendes oder etwas zukünftig Vorgestelltes.
} 
Future Skills beziehen sich also (1) gemäß der Zeit- oder Subjektdimension entweder auf individuell entwicklungsbezogene Aspekte des handelnden Subjekts (beispielsweise die Fähigkeit zur Selbstreflexion in Bezug auf etwas in der Vergangenheit Erlebtes oder ethische Kompetenz) oder beziehen sich (2) auf den Umgang mit einem Gegenstand, einem Objekt, etwa einem Thema oder einer Aufgabe (beispielsweise Design Thinking Skills), oder aber (3) auf die soziale Umwelt oder die Organisation, in der das Individuum handelt (beispielsweise Kooperations- oder Kommunikationskompetenzen). Subjekt, Objekt oder Welt/Organisationsbezug spannen also die Kompetenzfelder auf, in denen sich Future Skills verorten lassen. Abb. 3 zeigt die Aufgliederung der Future Skills in die unterschiedlichen Kompetenzfelder.

Alle drei Dimensionen stehen wiederum miteinander in Verbindung und beeinflussen sich wechselseitig. So wirkt beispielsweise die Kompetenz zur Selbstreflexion nicht nur auf die subjektive Entwicklung eines handelnden Individuums, sondern auch auf die Kommunikations- und Kooperationsfähigkeit (soziale oder Organisationsdimension) und wiederum auf die Systemkompetenz eines Individuums (Objektdimension). Insofern sind an jeder Handlung unterschiedliche Future Skills gleichermaßen beteiligt. Die drei Dimensionen formen so die Future-Skill-Triple-Helix-DNA, in der die drei Skill-Dimensionen bei konkreten Handlungen zusammenwirken (siehe Abb. 4). Sie ermöglichen ein besseres Verständnis der Faktoren, die zukünftige Handlungsfähigkeit ausmachen.

\section{$5 \quad$ Wandel von Lebens- und Arbeitskontexten}

Das Rückführen der Future Skills auf drei konstitutive Komponenten erlaubt auch eine Aufklärung der Ursachen, die Future Skills so bedeutsam machen. Die empirischen Analysen der Interviewdaten zeigen, dass in jeder der drei Dimensionen Veränderungsprozesse - im Folgenden als Shift bezeichnet - und Verschiebungen ablaufen. Dabei wird deutlich, dass sich ein klarer Wandel im Hinblick auf die Natur derjenigen Fähigkeiten abzeichnet, die für Individuen und deren Handlungsfähigkeit in zukünftigen Arbeits- und Lebenskontexten bedeutsam sind. Künftige Skill-Anforderungen können also von denen der Vergangenheit und auch zum Teil von den gegenwärtigen klar unterschieden werden. 


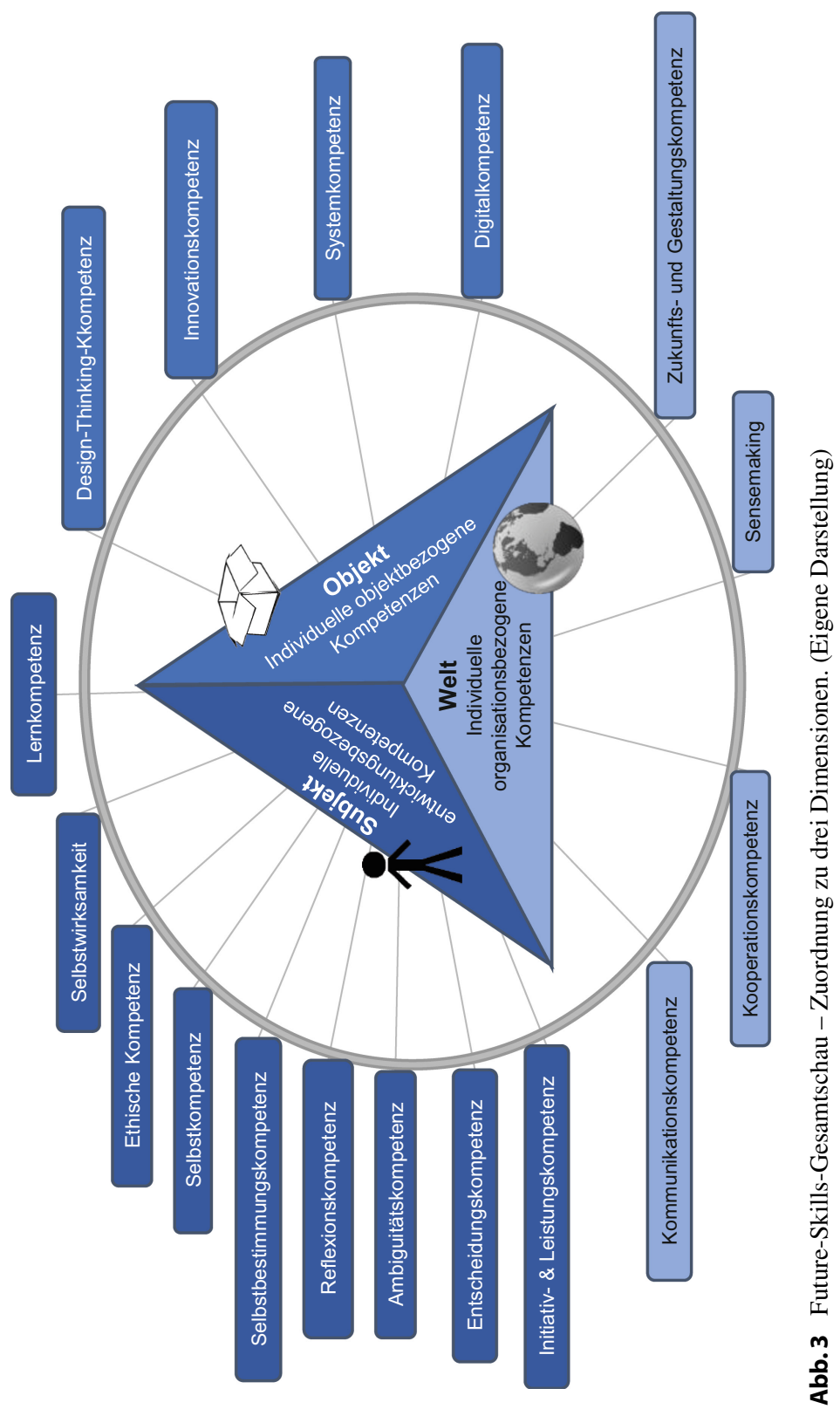


Abb. 4

Triple-Helix-Konzept der

Future Skills. (Eigene

Darstellung)

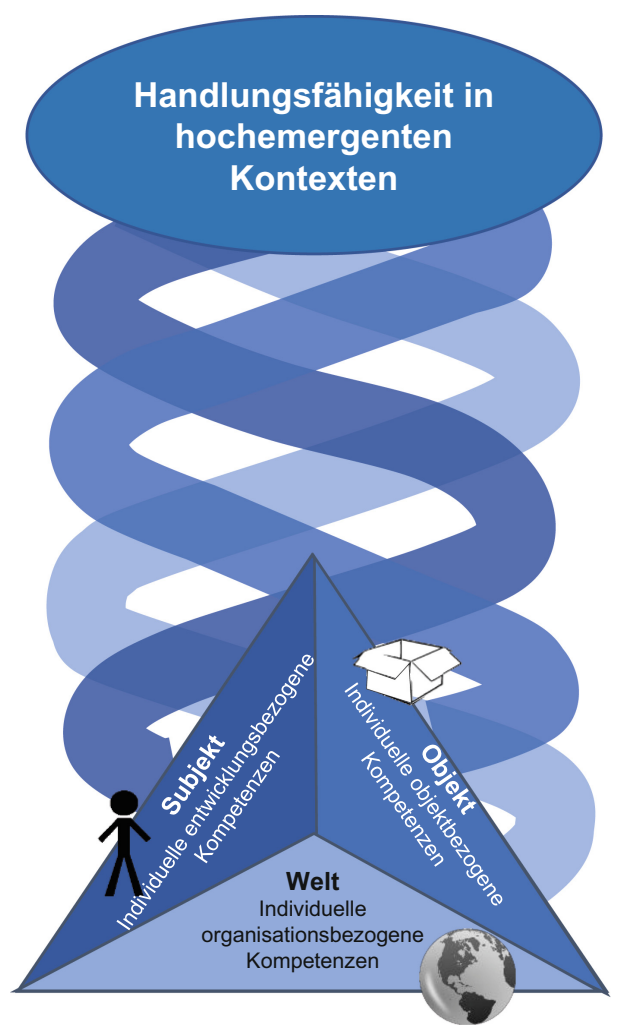

\subsection{Shift 1 - weniger Standardisierung und mehr Selbstorganisation}

Die Thesen von Voss und Pongratz (1998) zum Arbeitskraftunternehmer, von Hitzler und Honer zur Bastelbiographie (1994) und auch von Ulrich Beck (1986) zur Risikogesellschaft legt eine immer geringere Standardisierung von Erwerbsbiographien nahe. Daraus folgt eine stärkere Selbstkontrolle des Individuums in Bezug auf seine Erwerbsbiographie. Diese Selbstkontrolle der Navigation von einem Job zum nächsten, aber auch von einer Position innerhalb eines Berufes zur nächsten oder von einem Beruf zum nächsten zeigt sich in den 
Future-Skills-Daten auch auf einer Mikroebene. ${ }^{4}$ Auf dieser Ebene lassen sich für die Binnenanforderungen innerhalb von Arbeitsprozessen Fähigkeiten rekonstruieren, die nahelegen, dass es einen Wandel gibt, der weniger vorgegebene Arbeitsstrukturen und mehr Selbstorganisation erfordert. Diese Betonung von Selbstkompetenzen drückt sich in der Rekonstruktion der Daten aus, in denen die Befragten betonen, dass Handlungskontexte in Organisationen sich immer schneller wandeln, sowohl strukturell (in der Organisation) als auch inhaltlich (im Aufgabenbereich) und sozial (im Umfeld). Es wird deutlich, dass dabei die Individuen immer stärkere individuelle Anpassungsleistungen an neue Handlungskontexte erbringen müssen. Diese entstehen oftmals im Rahmen von emergenten Prozessen und sind nur schwer plan- oder vorhersehbar. Die notwendigen Fähigkeiten, die als Future Skills von den Befragten gefordert werden, haben die Aufgabe, diese Anpassungsleistung zu ermöglichen. Dabei wird deutlich, dass ein produktiv-antizipierender Umgang mit sich wandelnden Handlungskontexten eine immer stärkere Bedeutung erfährt, damit nicht kompensatorische Maßnahmen im Vordergrund stehen, die etwa bei Verlust der Handlungsfähigkeit aufgrund von sich ändernden Handlungskontexten darauf abzielen, Handlungsfähigkeit wiederherzustellen. Vielmehr geht es darum, Akteur*innen zu befähigen, bereits im Verlauf der Änderungsprozesse neue Handlungskontexte produktiv mitzugestalten. Future Skills haben dabei die Aufgabe, Akteur*innen zu befähigen, selbst organisiert handlungsfähig zu sein. Sogenannte Selbstkompetenzen wie beispielsweise Selbstwirksamkeit, Selbstbestimmung, Selbstkompetenz, Reflexionskompetenz und auch das selbst gesteuerte Lernen ermöglichen es den Individuen, die notwendigen Anpassungsvorgänge in hochemergenten Kontexten produktiv zu leisten.

\footnotetext{
${ }^{4}$ Nachtwey (2016) beschreibt den Wandel auf dem Arbeitsmarkt folgendermaßen: Für die Arbeitnehmer*innen war mit dem Normalarbeitsverhältnis Vorhersehbarkeit des Lebensverlaufes und relative soziale Sicherheit verbunden. Lediglich circa $10 \%$ der Beschäftigten arbeiteten zu Beginn der 1970er-Jahre in Teilzeit. Dominierte in den 1970er-Jahren noch das sichere Normalarbeitsverhältnis die ökonomische Szenerie, so sind in Deutschland im Jahr 2011 lediglich $28 \%$ der westdeutschen Beschäftigten in privatwirtschaftlichen Firmen mit Branchentarifverträgen beschäftigt (Gundert und Hohendanner 2011). Im Jahr 1998 waren es noch $39 \%$ (ebenda). Zudem hat sich in einzelnen Branchen das Leiharbeitsverhältnis von der Ausnahme zur Regel gewandelt. In der Lebensmittelindustrie besitzt in Deutschland zurzeit nur jede und jeder zehnte Beschäftigte einen regulären Arbeitsvertrag (ebenda). Aber auch im Segment der Höherqualifizierten dreht sich die Deregulierungsspirale mit Auswirkungen für die Beschäftigten. Gerade bei IT-Spezialistinnen und -spezialisten nehmen die Werkverträge zu und die Praxis des Crowdworkings verdrängt in der Software- und Autoindustrie fest definierte Tätigkeiten immer häufiger (ebenda).
} 


\subsection{Shift 2 - vom Fachwissen zur Handlungskompetenz}

Ein zweiter Shift, der sich aus den Interviewdaten ergibt, ist der Wandel von der ursprünglich hohen Bedeutung des Fachwissens hin zu einer eher als generisch beschriebenen Handlungskompetenz. Dabei fassen wir in Anlehnung an Erpenbeck (2012) Handlungskompetenz grundsätzlich als die Disposition zur zielgerichteten Handlungsfähigkeit in komplexen und unbekannten Problemsituationen. Es stehen vier Dimensionen im Mittelpunkt, die den hier beschriebenen Shift gut veranschaulichen können. Die ursprünglich für den Bereich der Medienkompetenz und des Umgangs mit Medien entwickelten Kompetenzdimensionen (in Anlehnung an Baake, zitiert nach Vollbrecht 2001, S. 56) werden dabei allgemein auf Handlungsfähigkeit in emergenten Kontexten bezogen, da sich an ihnen der Shift auch im Kompetenzverständnis gut veranschaulichen lässt:

- Die Wissensdimension mit einer informativen und einer instrumentellqualifikatorischen Dimension,

- die Dimension der Wissensanwendung mit einer eher rezeptiven und einer eher interaktiven Komponente,

- die Gestaltung von Neuem mit einer innovativen und einer kreativen Komponente und

- die Kritikfähigkeit in Bezug auf einen Wissensbestand mit einer analytischen, einer reflexiven (hier selbstbezogenen) und einer ethischen Komponente.

Über die Erkenntnis hinaus, dass Future Skills eher Handlungskompetenz verlangen und nicht mehr nur reines Fachwissen ausreicht, erlaubt es das Modell, wesentlich präziser zu rekonstruieren, welche Dimensionen von Kompetenz im Future-Skills-Modell ausgeprägt sind. Dabei wird in den Interviews deutlich darauf hingewiesen, dass Future Skills vor allem die Entwicklung der Gestaltungsund der kritischen Dimension von Kompetenz notwendig machen. Individuen konnten sich in der Vergangenheit darauf beschränken, Wissen, Methoden und Tools anzuwenden; künftig wird es aber zunehmend wichtiger werden, neues Wissen, Methoden und Tools originell und auf kreative Art und Weise zu entwickeln. 


\subsection{Shift 3 - von hierarchischen zu vernetzten Organisationskontexten}

Ein dritter Wandel bezieht sich auf ein sich generell wandelndes Organisationsumfeld von hierarchischen Ablauforganisationen hin zu vernetzten und agilen Organisationen. Der sich hier vollziehende Wandel wird in den Interviewdaten deutlich beschrieben: Während Organisationen in der Vergangenheit in klaren Strukturen und Managementprozessen organisiert waren, werden die Organisationen der Zukunft in fluideren Strukturen organisiert werden, die schnelleren und grundsätzlicheren Änderungen unterliegen. Dabei stehen konkurrierende Pole einander gegenüber, bei denen die bisherigen Strukturen und Abläufe von klar definierten Managementstrukturen zukünftig eher durch agile Abläufe und ein Ermöglichungsmanagement ersetzt werden. Die Ablauforganisation wird in Zukunft durch vernetzte Strukturen geprägt sein, in denen klar definierte Prozesse sich häufiger weiterentwickeln und Organigramme und Zuständigkeiten sich schneller wandeln. Beziehungsmanagement wird dabei ein zunehmend wichtiger Faktor. Der gesamte Bereich informeller Eigeninitiative ist ein wichtiger Bestandteil von organisationalem Erfolg und ein wesentlicher Future Skill, ohne den die Steuerung von Organisationen zukünftig ineffizient wird. Die Befragten drücken aus, dass in Future Organisations zentrale Steuerungsansätze immer weniger zielführend sind und stattdessen beteiligungsorientierte Zielfindungsprozesse immer mehr an Bedeutung zunehmen.

\section{$6 \quad$ Fazit}

Im Hinblick auf Future Skills lassen sich folgende Punkte zusammenfassen:

1. Future Skills können analysiert und anhand eines Profilsets beschrieben werden, welches die 17 Skills in drei Dimensionen kategorisiert. Jede dieser Dimensionen enthält eine Reihe von Future-Skill-Profilen.

2. Diese Skills können durch zwei Eckpfeiler-Charakteristika beschrieben werden: eine starke, transversale und gut ausgebildete Fähigkeit zur Selbstorganisation, die mit der Fähigkeit einhergeht, in unvorhersehbaren Kontexten zu agieren. Diese beiden Elemente avancieren damit zu Schlüsselbestandteilen für Professionalität - unabhängig vom jeweiligen Berufsfeld.

3. Future Skills können mit einem Modell beschrieben werden, welches die 17 Skills anhand von drei Dimensionen kategorisiert: subjektive - individuelle entwicklungsbezogene Skills, objektive - aufgaben- und themenbezogene 
Skills, soziale - welt-/organisationsbezogene Skills. Alle drei Dimensionen stehen miteinander in Zusammenhang und sind daher nicht als bloßer Ausdruck isolierter Skill-Felder zu denken.

4. Der Future-Skill-Ansatz, wie er hier vorgestellt wurde, geht über ein statisches Modell der reinen Skill-Aufzählung und -definition hinaus. Außerdem geht das Modell zwar davon aus, dass digitale oder technische Skills künftig zweifelsohne eine wichtige Future-Skills-Zutat sein werden, sieht diese Skills aber nicht als allein ausreichend an. Der wirkliche Wert dieser Skills liegt daher vor allem in der persönlichen Entwicklung von Dispositionen, die das Individuum zu selbst organisiertem Handeln in einer definierten Domäne befähigen können.

\section{Literatur}

Beck, U. (1986). Die Risikogesellschaft. Frankfurt a. M.: Suhrkamp.

Brown, J. (2018). How these humanities graduates are finding jobs in silicon valley. PBS. https://www.pbs.org/newshour/show/how-these-humanities-graduates-are-findingjobs-in-silicon-valley\#transcript. Zugegriffen: 21. Okt. 2020.

Dewey, J., \& Bentley, A. (1949). Kñowing and the known. Boston: Beacon.

Ehlers, U.-D. (2020a). Future Skills: Lernen der Zukunft - Hochschule der Zukunft. Wiesbaden: Springer VS.

Ehlers, U.-D. (2020b). NextSkills. Future skills - The future of learning and higher education. https://nextskills.org/. Zugegriffen: 21. Okt. 2020.

Ehlers, U.-D., \& Kellermann, S. A. (2019). Future skills. The future of learning and higher education. Results of the international future skills delphi survey. https://nextskills.org/lib rary/future-skills-study/. Zugegriffen: 21. Okt. 2020.

Erpenbeck, J. (2012). Führungskompetenz. In W. G. Faix (Hrsg.), Kompetenz. Festschrift Prof. Dr. John Erpenbeck zum 70. Geburtstag, 4, (S. 109-142). Stuttgart: Steinbeis-Edition.

Gundert, S., \& Hohendanner, C. (2011). Leiharbeit und befristete Beschäftigung: Soziale Teilhabe ist eine Frage von stabilen Jobs. IAB-Kurzbericht, 4. Nürnberg.

Hachmeister, C.-D. (2017). Die Vielfalt der Studiengänge. Entwicklung des Studienangebotes in Deutschland zwischen 2014 und 2017. Gütersloh: CHE Centrum für Hochschulentwicklung. https://www.che.de/wp-content/uploads/upload/Im_Blickpunkt_Die_Vielfalt_ der_Studiengaenge_2017.pdf. Zugegriffen: 21. Okt. 2020.

Hartley, S. (2016). The fuzzy and the techie. Why the liberal arts will rule the digital world. Financial Times, (18. November). https://www.ft.com/content/e25235dc-aa8a11e6-9cb3-bb8207902122. Zugegriffen: 21. Okt. 2020.

Hippler, H. (2016). Wozu (noch) Geisteswissenschaften? Rotary Magazin, (03. Mai). https:// rotary.de/bildung/wozu-noch-geisteswissenschaften-a-8984.html. Zugegriffen: 21. Okt. 2020 . 
Hitzler, R., \& Honer, A. (1994). Bastelexistenz. Über subjektive Konsequenzen der Individualisierung. In U. Beck \& E. Beck-Gernsheim (Hrsg.), Riskante Freiheiten. Individualisierung in modernen Gesellschaften (S. 307-314). Frankfurt a. M.: Suhrkamp.

Kummert, T. (2017). Endlich einer, der nicht nur Formeln anwenden kann. Süddeutsche Zeitung, (20. August). https://www.sueddeutsche.de/karriere/arbeitsmarkt-endlich-einerder-nicht-nur-formeln-anwenden-kann-1.3623308. Zugegriffen: 21. Okt. 2020.

Mayring, P. (1996). Einführung in die Qualitative Sozialforschung. Eine Anleitung zu qualitativem Denken (3. Aufl.). Weinheim: Psychologie Verlags Union.

Meder, N. (2007). Der Lernprozess als performante Korrelation von Einzelnem und kultureller Welt. Eine bildungstheoretische Explikation des Begriffs. Spektrum Freizeit, 07, $I \& I I$ (S. 119-135). https://duepublico.uni-duisburg-essen.de/servlets/DerivateServlet/ Derivate-34931/21_Meder.pdf. Zugegriffen: 21. Okt. 2020.

Nachtwey, O. (2016). Die Abstiegsgesellschaft - Über das Aufbegehren in der Regressiven Moderne. Berlin: Suhrkamp.

Olejarz, J. M. (2017). Liberal arts in the data age. Harvard Business Review. https://hbr.org/ 2017/07/liberal-arts-in-the-data-age. Zugegriffen: 21. Okt. 2020.

Placke, B., \& Schleiermacher, T. (2018). Anforderungen der digitalen Arbeitswelt. Kompetenzen und digitale Bildung in einer Arbeitswelt 4.0. Auftraggeber: Bundesverband der Personalmanager e. V. Köln: IW Consult GmbH. https://www.iwkoeln.de/fileadmin/ user_upload/Studien/Gutachten/PDF/2018/Gutachten_Anforderungen_Digitale_Arbeits welt.pdf. Zugegriffen: 21. Okt. 2020.

Roth, H. (1971). Pädagogische Anthropologie. 2: Entwicklung und Erziehung. Hannover: Hermann Schroedel Verlag.

Thomas, D. R. (2006). A General Inductive Approach for Analyzing Qualitative Evaluation Data. American Journal of Evaluation, 27, 237-246.

Vollbrecht, R. (2001). Einführung in die Medienpädagogik. Weinheim: Beltz.

Voß, G., \& Pongratz, H. (1998). Der Arbeitskraftunternehmer. Eine neue Grundform der Ware Arbeitskraft? Kölner Zeitschrift für Soziologie und Sozialpsychologie, 50, 131-158.

Open Access Dieses Kapitel wird unter der Creative Commons Namensnennung 4.0 International Lizenz (http://creativecommons.org/licenses/by/4.0/deed.de) veröffentlicht, welche die Nutzung, Vervielfältigung, Bearbeitung, Verbreitung und Wiedergabe in jeglichem Medium und Format erlaubt, sofern Sie den/die ursprünglichen Autor(en) und die Quelle ordnungsgemäß nennen, einen Link zur Creative Commons Lizenz beifügen und angeben, ob Änderungen vorgenommen wurden.

Die in diesem Kapitel enthaltenen Bilder und sonstiges Drittmaterial unterliegen ebenfalls der genannten Creative Commons Lizenz, sofern sich aus der Abbildungslegende nichts anderes ergibt. Sofern das betreffende Material nicht unter der genannten Creative Commons Lizenz steht und die betreffende Handlung nicht nach gesetzlichen Vorschriften erlaubt ist, ist für die oben aufgeführten Weiterverwendungen des Materials die Einwilligung des jeweiligen Rechteinhabers einzuholen.

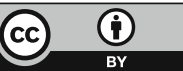

